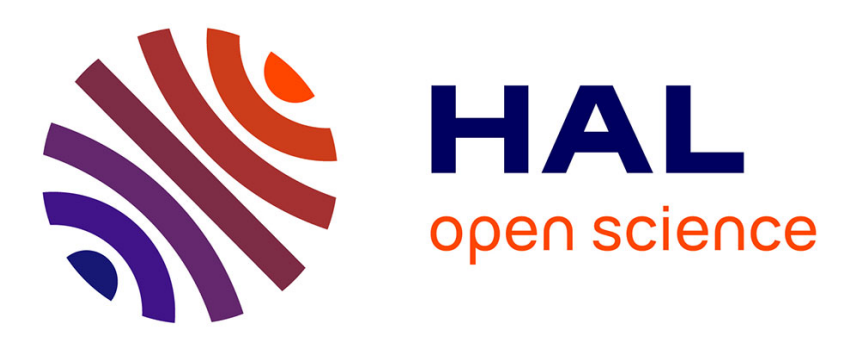

\title{
Out-of-Equilibrium Body Potential Measurements in Pseudo-MOSFET for Sensing Applications
}

Licinius Benea, Maryline Bawedin, Cécile Delacour, Irina Ionica

\section{To cite this version:}

Licinius Benea, Maryline Bawedin, Cécile Delacour, Irina Ionica. Out-of-Equilibrium Body Potential Measurements in Pseudo-MOSFET for Sensing Applications. Solid-State Electronics, 2018, 143, pp.69-76. 10.1016/j.sse.2017.11.010 . hal-01685404

\section{HAL Id: hal-01685404 https://hal.science/hal-01685404}

Submitted on 16 Jan 2018

HAL is a multi-disciplinary open access archive for the deposit and dissemination of scientific research documents, whether they are published or not. The documents may come from teaching and research institutions in France or abroad, or from public or private research centers.
L'archive ouverte pluridisciplinaire HAL, est destinée au dépôt et à la diffusion de documents scientifiques de niveau recherche, publiés ou non, émanant des établissements d'enseignement et de recherche français ou étrangers, des laboratoires publics ou privés. 


\title{
Out-of-Equilibrium Body Potential Measurements in Pseudo-MOSFET for Sensing Applications
}

\author{
Licinius Benea $^{1}$, Maryline Bawedin ${ }^{1}$, Cécile Delacour ${ }^{2}$, Irina Ionica ${ }^{1}$ \\ ${ }^{1}$ Univ. Grenoble Alpes, CNRS, Grenoble INP, IMEP-LAHC 38016 Grenoble, France \\ ${ }^{2}$ Néel Inst., CNRS, 38042 Grenoble, France
}

\begin{abstract}
The aim of this paper is to present the out-of-equilibrium body potential behaviour in the $\Psi$-MOSFET configuration. Consistent measurements in this experimental setup succeeded in providing a substantial understanding of its characteristics in the depletion region. The final objective of this work is to envision this new measurement technique for biochemical sensor applications. Among its advantages, the most important are its simplicity, the good sensitivity, the measurement of a potential instead of a current and the low bias needed for detection compared to the conventional drain current measurements.
\end{abstract}

Keywords: SOI, $\Psi$-MOSFET, body potential, biochemical sensor, field-effect

\section{Introduction}

The $\Psi$-MOSFET is a characterization technique used for bare silicon on insulator wafers to determine important material parameters such as the mobility and the interface trap density [1]-[4]. This approach uses the innate upsidedown structure of a SOI substrate, where the bulk silicon substrate is used as a back-gate (named simply "gate") and the buried oxide $(\mathrm{BOX})$ as a gate dielectric. The gate voltage $\left(\mathrm{V}_{\mathrm{G}}\right)$ induces a channel at the interface between the top silicon film and the BOX. The drain current $\left(\mathrm{I}_{\mathrm{D}}\right)$ is measured between two metallic probes placed on the silicon film, which play the roles of source and drain. For low doping concentrations of silicon films (i.e. $10^{15} \mathrm{~cm}^{-3}$ ), the conduction can be ensured by holes or electrons depending on the polarity of $\mathrm{V}_{\mathrm{G}}$. The coupling between the top free surface charge and the channel leads to threshold/flat band voltage shifts and was first observed during the electrical characterization of $\Psi$-MOSFET with different states induced at the top surface[4]. For intentionally modified SOI surfaces, these $\mathrm{V}_{\mathrm{T}} / \mathrm{V}_{\mathrm{FB}}$ shifts proved to be useful for chemical detection [5], making the $\Psi$-MOSFET a detection device. Due to the fact that the channel is very close to the surface, this device has a high sensitivity, especially for the thin silicon films.

This paper aims to propose an alternative detection method, by measuring the body potential rather than the conventionally used drain current. It derives from the body-potential measurements in the out-of-equilibrium regime already performed on SOI-based MOSFETs [6]. In the case of an nMOSFET, if the gate is quickly polarized to induce the inversion regime, an out-of-equilibrium state is generated by the excess of majority carriers (holes) in the channel, which produces a body potential increase. An analogous behaviour can be observed for pMOSFET transistors. The use of a potential measurement instead of a current one for detection ensures the good sensitivity and a lower bias needed for sensing.

We will present the materials and the methods in section 2, the body potential measurements, their dependence on different electrical test parameters such as the scanning speed and their significance are presented in section 3 . The invariability to probe pressure and position are an asset for detection (as described in section 4). Section 5 shows an example of detection of charged gold nanoparticles.

\section{Materials and methods}

The measurements presented in this paper were realized on devices built on silicon on insulator (SOI) substrates which had a $145 \mathrm{~nm}$ thick buried oxide (BOX) and an $88 \mathrm{~nm}$ silicon film. The $\Psi$-MOSFET structures are $5 \times 5 \mathrm{~mm}^{2}$ islands patterned by photolithography and Reactive Ion Etching (RIE).

The electrical measurements were conducted using an HP4155 Analyser. The setup consists in three tungsten carbide probes separated by 1 millimetre and having a pressure controlled system (Figure 1). The probes number 1 and 3 play the role of source and drain, respectively, whereas the probe number 2 is used to measure the body potential. 


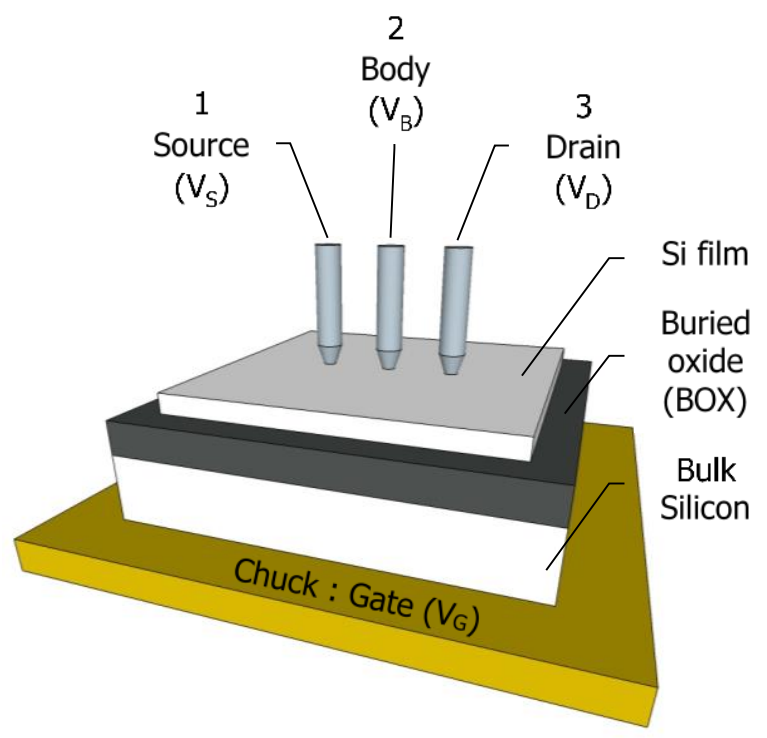

Figure 1: Experimental setup for body-potential measurements in the $\Psi$-MOSFET configuration.

For potential measurements, two possibilities are available on the HP4155 Analyser: a Voltage Monitor Unit (VMU) or a Source/Monitor Unit (SMU) used in a current source mode by imposing a zero current condition. Both configurations were used to measure the same sample, in the aim of determining the most appropriate one. Figure 2 traces the $\mathrm{I}_{\mathrm{G}}$ current and the opposite of $\mathrm{I}_{\mathrm{D}}+\mathrm{I}_{\mathrm{S}}$. In the absence of a leakage current through the measurement system, the sum of the all currents through the device should be equal to 0 , so $I_{G}=-\left(I_{D}+I_{S}\right)$. This is actually the case for SMU (Figure 2a). In the case of the VMU (Figure 2b), a leakage current of $0.8 \mathrm{nA}$ was observed. This is consistent with the $\sim 1$ nA leakage current announced by the HP4155 manufacturer for VMU measurements [7]. The $-\left(\mathrm{I}_{\mathrm{D}}+\mathrm{I}_{\mathrm{S}}\right)$ curve presents important fluctuations for $\mathrm{V}_{\mathrm{G}}>2 \mathrm{~V}$, which is not the case for the $\mathrm{I}_{\mathrm{G}}$ curve. This is probably related to the fact that $I_{D}$ and $I_{S}$ are at least 3 orders of magnitude higher than $I_{G}$, the difference in range means that the resolutions are not the same.
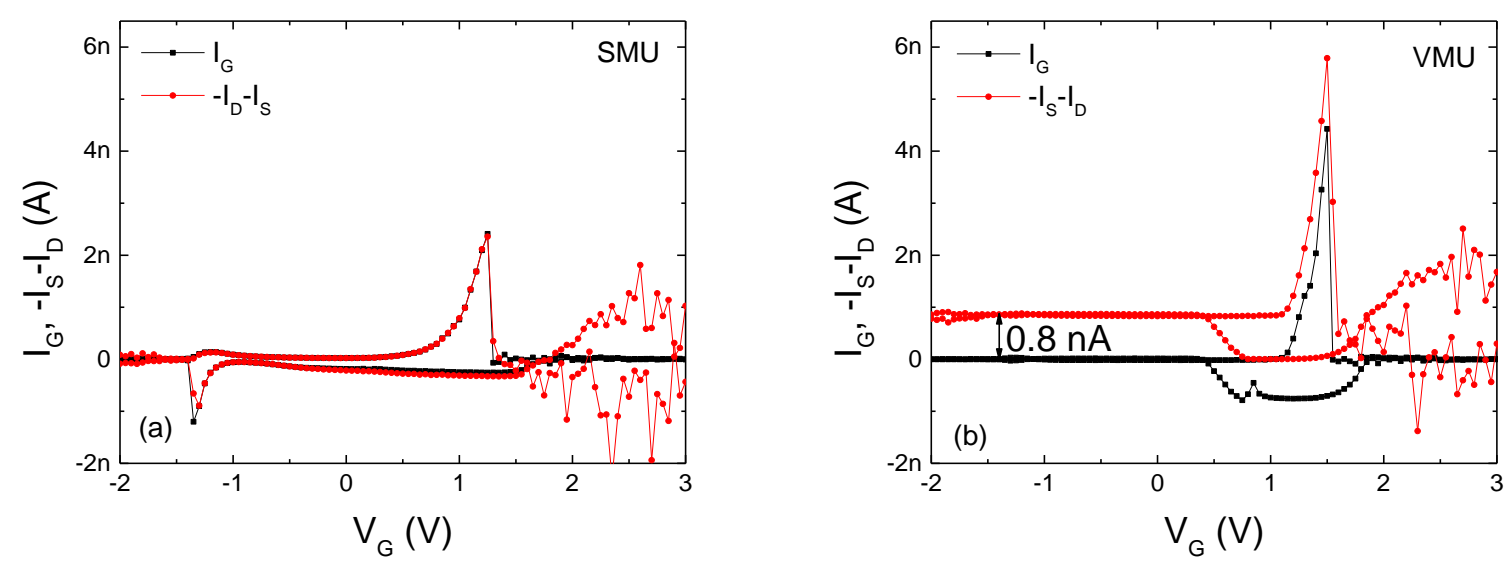

Figure 2: Gate current and the opposite of the sum of drain and source currents vs. gate bias. Body potential measured with SMU (a) or VMU (b). A leakage current of $0.8 n A$ is observed when performing the measurement with VMU. 3 probe measurement, $V_{D}=0.1 \mathrm{~V}, V_{G}$ step $50 \mathrm{mV}$, probe pressure $100 \mathrm{~g}$.

This measurement setup was further validated, as required for high impedance DUTs, by comparing the potential obtained under a zero current condition $(\mathrm{I}=0 \mathrm{~A})$ and a very small $(\mathrm{I}=10 \mathrm{pA})$ current imposed through the SMU used to measure $\mathrm{V}_{\mathrm{B}}$. The values obtained for the body potential were perfectly matched, which allowed us to conclude that the correct method to measure the body potential is to use a SMU in a zero current condition. Additionally, the input impedance is $\geq 10^{15} \Omega$ for the SMU and $\geq 1 \mathrm{G} \Omega$ for the VMU, which makes the SMU more appropriate for $\mathrm{V}_{\mathrm{B}}$ measurements. 


\section{Body potential measurements - relationship between $I_{D}, V_{B}, I_{G}$}

Figure 3 presents the three main parameters of the body potential method, namely the drain current $\left(\mathrm{I}_{\mathrm{D}}\right)$, the body potential $\left(\mathrm{V}_{\mathrm{B}}\right)$ and the gate current $\left(\mathrm{I}_{\mathrm{G}}\right)$ for different sweeping directions of the gate bias - from accumulation to inversion (Forward) and from inversion to accumulation (Reverse). The body potential probe that stands halfway between the source and the drain. According to the tension divider bridge principle, in the accumulation and in the inversion regimes, the value of $V_{B}$ is equal to half of $V_{D}$.

The body potential variation takes place in the depletion region, where the drain current is negligible and where the passage from a channel of holes to a channel of electrons (or the opposite) is imposed by the $V_{G}$ variation. When the gate bias is scanned from accumulation to inversion (Figure 3a), the body potential increases with a quasi-linear behaviour, following $\mathrm{V}_{\mathrm{G}}$, and then sharply decreases when the inversion channel begins to form.

An equivalent response can be observed when the gate is scanned from inversion to accumulation (Figure $3 b$ ). However, due to the fact that the carriers involved are of opposite polarities, the body potential has negative values in this case. The same polarity change is observed for the $\mathrm{I}_{\mathrm{G}}$ peak, its significance will be discussed in the followings. Note additionally that the $I_{D}$ values in this region are negative (empty black squares) or positive (filled black squares) depending on the sweeping direction.

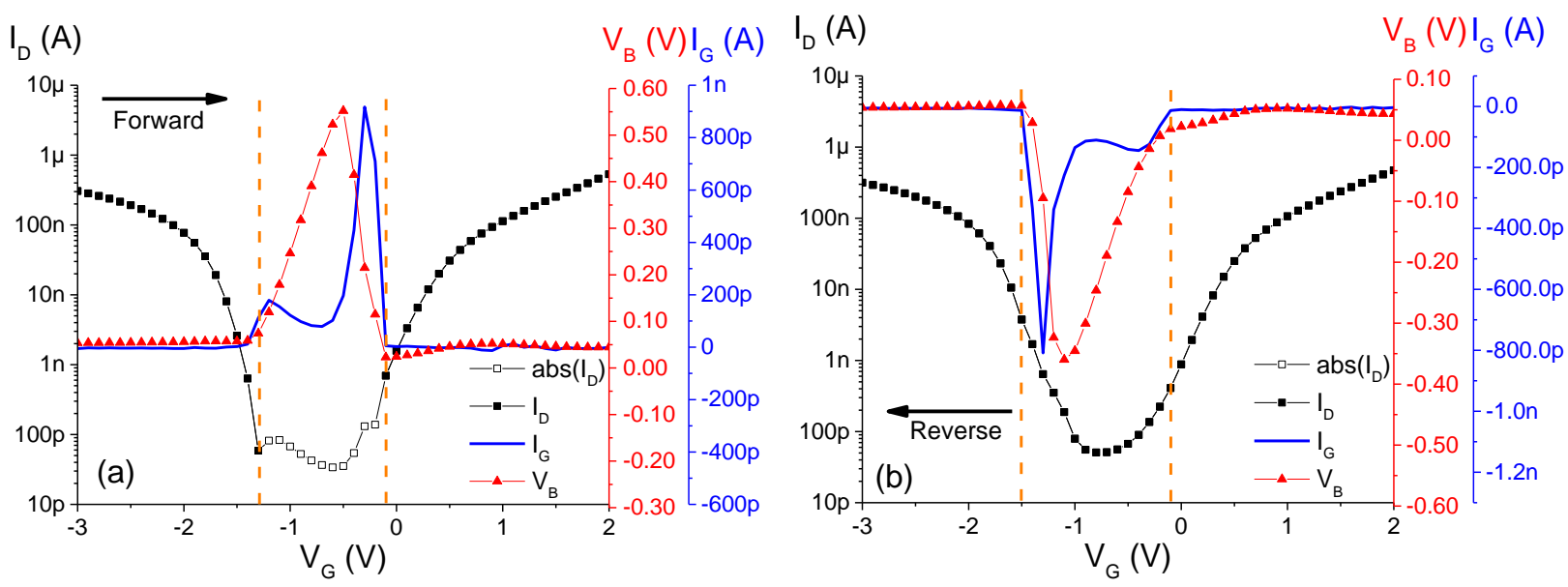

Figure 3: Drain current, body potential and gate current vs. gate voltage sweep from accumulation to inversion (a) and from inversion to accumulation $(b)$. All curves were measured simultaneously. 3 probe measurement, $V_{D}=0.1 \mathrm{~V}$, $V_{G}$ step $100 \mathrm{mV}$, probe pressure $100 \mathrm{~g}$.

Significant variations of $\mathrm{V}_{\mathrm{B}}$ are associated with corresponding variations of $\mathrm{I}_{\mathrm{G}}$. This particular behaviour appears when switching from accumulation to inversion and vice versa.

An important proof that the $I_{G}$ peak is related to the variations of $V_{B}$ is provided by the derivative of $V_{B}$ with respect to $V_{G}$ (Figure $4 \mathrm{a}$ and $\mathrm{b}$ ). The $\mathrm{dV}_{\mathrm{B}} / \mathrm{dV}_{\mathrm{G}}$ curve follows the same trend as $\mathrm{I}_{\mathrm{G}}$ regarding the peak. These terms are related via a capacitance coefficient $(\mathrm{C})$, as documented in [6]:

$$
\frac{I_{G}}{C}=\frac{\Delta\left(V_{G}-V_{B}\right)}{\Delta t} \Leftrightarrow \frac{\Delta V_{B}}{\Delta V_{G}}=1-\frac{I_{G}}{C} \cdot \frac{\Delta \mathrm{t}}{\Delta V_{G}}
$$

The direct relationship between the out-of-equilibrium body potential and the formation of the accumulation/inversion layer is thus confirmed. 

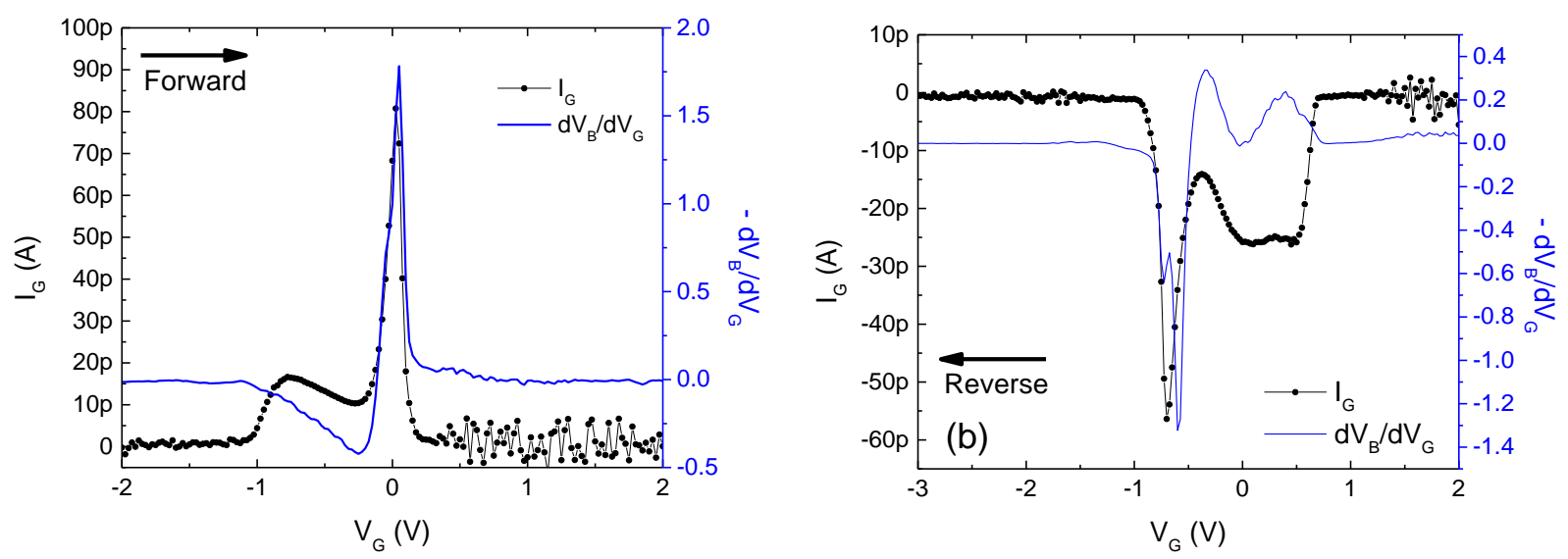

Figure 4: Gate current and $d V_{B} / d V_{G}$ vs. gate voltage sweep from accumulation to inversion (a) and from inversion to accumulation (b). The two peaks correspond in both cases, which probes the direct connection between $V_{B}$ and $I_{G} .3$ probe measurement, $V_{D}=0.1 \mathrm{~V}$, $V_{G}$ step $25 \mathrm{mV}$, probe pressure $100 \mathrm{~g}$.

As previously mentioned, the body potential follows the behaviour of the gate bias, and this regardless of the starting point of the measurement (Figure 5 a to d). When starting the measurement in the depletion region, without a hold time (time imposed for the settlement of the initial equilibrium conditions of the measurement), the body potential directly follows the gate bias (Figure $5 \mathrm{a}$ and $\mathrm{c}$ ). The return to equilibrium (when $\mathrm{V}_{\mathrm{B}}$ drops), is not modified significantly, which is confirmed by the unchanged $\mathrm{I}_{\mathrm{G}}$ peak (Figure $5 \mathrm{~b}$ and $\mathrm{d}$ ).
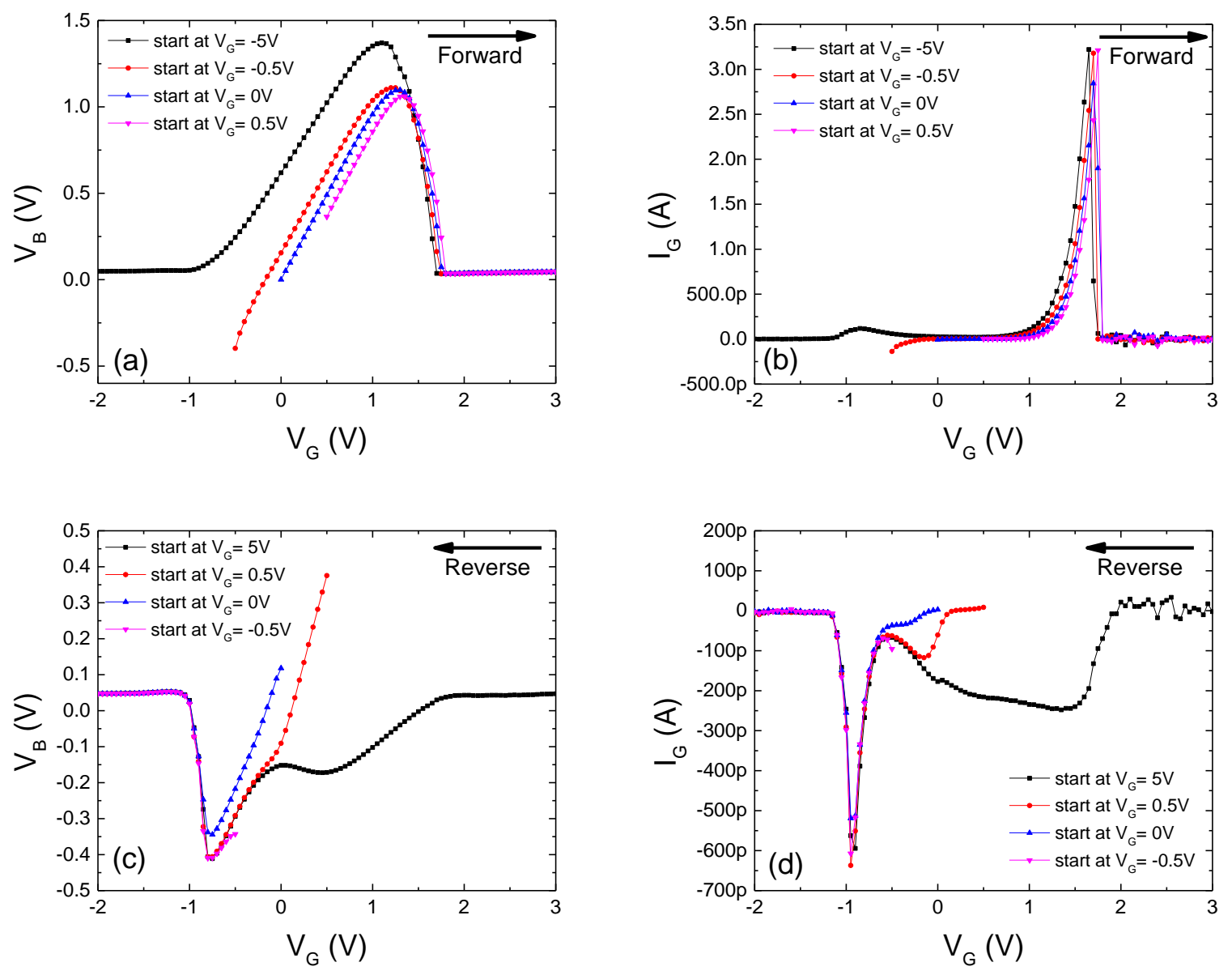
Figure 5: Body potential vs. gate voltage $(a)$ and $(c)$ and gate current vs. gate voltage $(b)$ and (d) for different starting points and for different sweeping directions. The body potential follows the gate bias in the depletion region. 3 probe measurement,

$$
V_{D}=0.1 \mathrm{~V} \text {, V } V_{G} \text { step } 50 \mathrm{mV} \text {, probe pressure } 100 \mathrm{~g} \text {. }
$$

Note that the values of the body potential and of the gate current in Figure 3 and Figure 5 differ, especially in the case of $V_{B}$ in the forward sweeping direction. For nanoindentation-based measurements, it is necessary to consider changes in the tip geometry with time. As reported in [8], it was found that the Berkovich diamond indenter tips wear significantly when indenting hard materials such as silicon. The same phaenomenon applies in the case of the WC probes used here. The blunting of the tip reduces the tip penetration depth and the contact area, which translates into a change of the contact resistance. However, our results suggest that variations in the tip pressure does not affect significantly the $\mathrm{V}_{B}$ dependence on $\mathrm{V}_{\mathrm{G}}$, and thus should rather stem from tip aging during a long period of time. In our case, the higher $\mathrm{V}_{\mathrm{B}}$ in Figure 5 is due to the fact that the measurements were made at a later stage than those in Figure 3. We mention that this is a long-term effect and that the increase of the body potential in time can be considered as a favourable consequence for sensing purposes.

The out-of-equilibrium body potential is the consequence of the time needed to evacuate the excess of carriers by recombination or drift-diffusion current conduction out of the body in the SOI-based devices. In spite of the direct relationship with the gate bias, $V_{B}$ is also highly dependent on the measurement time. In Figure $6 a$ and $b$, the transient behaviour of $\mathrm{V}_{\mathrm{B}}\left(\mathrm{V}_{\mathrm{G}}\right)$ and $\mathrm{I}_{\mathrm{G}}\left(\mathrm{V}_{\mathrm{G}}\right)$ are exposed for different measurement step biases. In this way, the ratio between the measurement times is approximately inversely proportional to the ratio between the steps.

It can be observed that faster sweeps (i.e. with a higher measurement step) induce higher peaks of $V_{B}$ as well as $I_{G}$. This reflects the time dependant characteristics of the body potential combined with capacitive coupling and highlights its out-of-equilibrium trait.
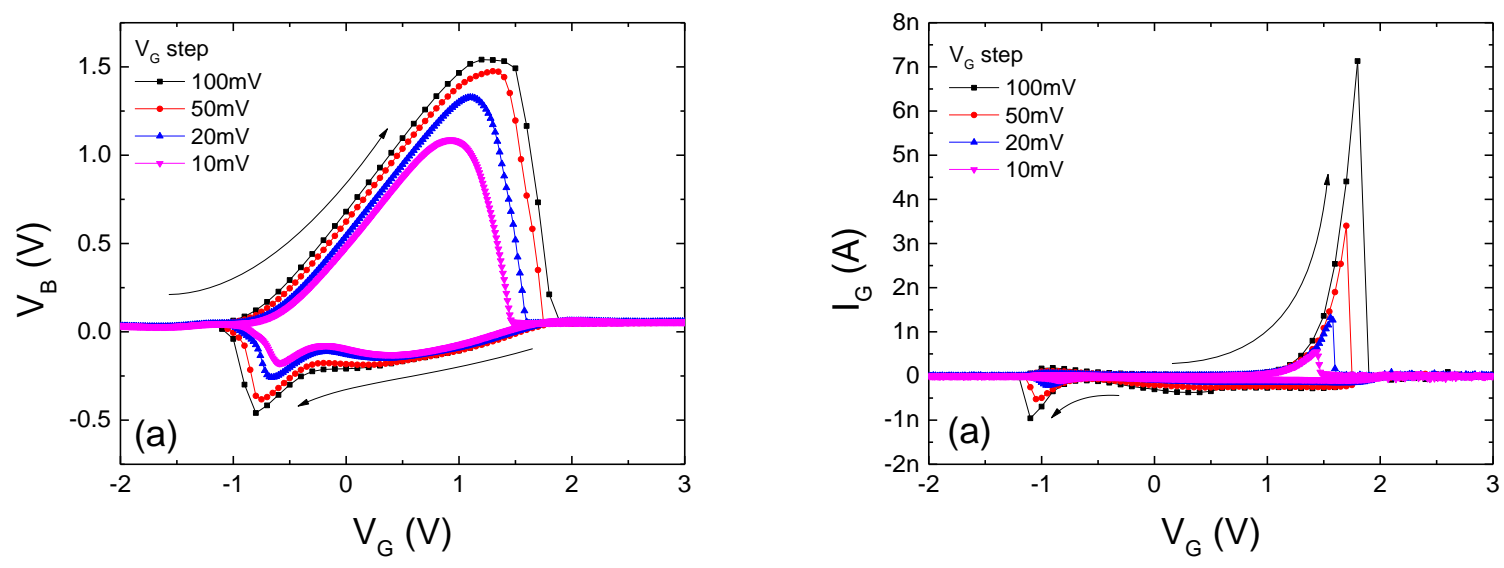

Figure 6: Body potential vs. gate voltage (a) and gate current vs. gate voltage $(b)$ for different measurement steps. Faster sweeps (higher measurement step) induce higher values of both $V_{B}$ and $I_{G} .3$ probe measurement, $V_{D}=0.1 \mathrm{~V}$, probe pressure $100 \mathrm{~g}$.

The $\mathrm{I}_{\mathrm{G}}$ peak occurs just before creating the channel of electrons (forward sweeping direction) or the channel of holes (reverse sweeping direction) as evidenced in Figure 3 by the $\mathrm{I}_{\mathrm{D}}$ current. Consequently, $\mathrm{I}_{\mathrm{G}}$ in this region is probably a displacement current responsible for the charge/discharge of the BOX capacitance. In order to verify this statement, an integral of $I_{G}$ over $V_{G}$ was determined for the different measurement steps. The injected charge $\left(Q_{G}\right)$ can be calculated by integrating $\mathrm{I}_{\mathrm{G}}$ over time:

$$
Q_{G}=\int I_{G} \cdot d t
$$

As the time is a parameter that is not directly available for the measurements, the gate bias had to be introduced in the calculation:

$$
Q_{G}=\int I_{G} \cdot d V_{G} \cdot \frac{d t}{d V_{G}}
$$


The $\frac{d t}{d V_{G}}$ term is a constant for each experiment and is proportional with the measurement time, thus inversely proportional with the measurement step. $\mathrm{I}_{\mathrm{G}}$ can then be integrated over $\mathrm{V}_{\mathrm{G}}$ in the $0 \mathrm{~V}$ to $2 \mathrm{~V}$ interval. The total charge injected needed to charge the BOX capacitance when switching from accumulation to inversion is always the same independently of the scanning speed $\left(\mathrm{Q}_{\mathrm{G}}\right.$ must be the same for all the curves in Figure 6b). The approximate measurement time is calculated after the $\frac{d t}{d V_{G}}$ term, which is inversely proportional to the measurement step and is presented in Table 1 as a ratio with respect to the $10 \mathrm{mV}$ curve (e.g. the measurement time of the $100 \mathrm{mV}$ curve is a tenth of the measurement time of the $10 \mathrm{mV}$ curve). Column 3 presents the integral $\int I_{G} \cdot d V_{G}$ over the [0V; $\left.2 \mathrm{~V}\right]$ interval. This quantity was also normalized with respect to the $10 \mathrm{mV}$ curve in column 4 . This amount of charges $\left(\mathrm{Q}_{\mathrm{G}}\right)$, measured in arbitrary units, was calculated then as the product between the relative measurement time with respect to the $10 \mathrm{mV}$ curve (column 2) and the relative value of the integral, also with respect to the $10 \mathrm{mV}$ curve (column 4). The obtained values are consistently close to 1 (column 5), with the exception of the $100 \mathrm{mV}$ case, which can be explained by its low resolution (greater distance between measuring points). It can be thus considered that the $\mathrm{I}_{\mathrm{G}}$ current peak corresponds to a displacement current. The same conclusion stands for the reverse sweeping direction (results not shown here). It is worth mentioning that this displacement current is probably limited by the carrier injection at the silicon film channel-BOX interface. This is mainly due to a lower value of the substrate resistance (approximately $3 \Omega$ for a $5 \times 5 \mathrm{~mm}^{2}$ die) compared to the series resistance between the probes and the silicon film (in the order of $10^{3} \Omega$ in the inversion regime and $10^{5} \Omega$ in the accumulation regime as extracted from $\mathrm{I}_{\mathrm{D}}-\mathrm{V}_{\mathrm{G}}$ measurements [9]).

Table 1: Values following the integration of $I_{G}$ over $V_{G}$ for different measurement steps

\begin{tabular}{ccccc}
\hline $\begin{array}{c}\text { Measurement } \\
\text { step }\end{array}$ & $\begin{array}{c}\text { Approximate } \\
\text { measurement } \\
\text { time ratio (/10mV) }\end{array}$ & $\begin{array}{c}\text { Integration } \\
(\mathbf{A} \cdot \mathbf{V}) \\
{[\mathbf{0 V} ; \mathbf{2 V}]}\end{array}$ & $\begin{array}{c}\text { Integration } \\
\text { ratio }(/ \mathbf{1 0 m}) \\
{[\mathbf{0 V} ; \mathbf{2 V}]}\end{array}$ & $\begin{array}{c}\text { QG (a.u.) } \\
{[\mathbf{0 V} ; 2 \mathbf{2 V}]}\end{array}$ \\
\hline $10 \mathrm{mV}$ & 1 & $1.38 * 10^{-10}$ & 1 & 1.00 \\
$20 \mathrm{mV}$ & 0.5 & $2.86 * 10^{-10}$ & 2.07 & 1.04 \\
$50 \mathrm{mV}$ & 0.2 & $6.99 * 10^{-10}$ & 5.07 & 1.01 \\
$100 \mathrm{mV}$ & 0.1 & $17.4 * 10^{-10}$ & 12.6 & 1.26 \\
\hline
\end{tabular}

\section{Experimental setup for biosensing - probe positioning and pressure invariability}

The ultimate goal of the out-of-equilibrium body potential measurements is its implementation in the biochemical sensing field. This implies the need for a liquid chamber placed on the top surface of the silicon film (Figure 7). In this regard, the position of the probes must be outside the liquid chamber.

The experimental results presented previously were obtained using a drain electrode which helped observing the formation of the inversion/accumulation layer by measuring the drain current at the same time as the body potential. The three probes, separated by $1 \mathrm{~mm}$, were placed in the middle of the silicon islands. For practical and simple sensing purposes, the out-of-equilibrium body potential measurements can be also performed using only two probes instead of three: one for measuring the body potential and the other as a reference electrode (grounded source).

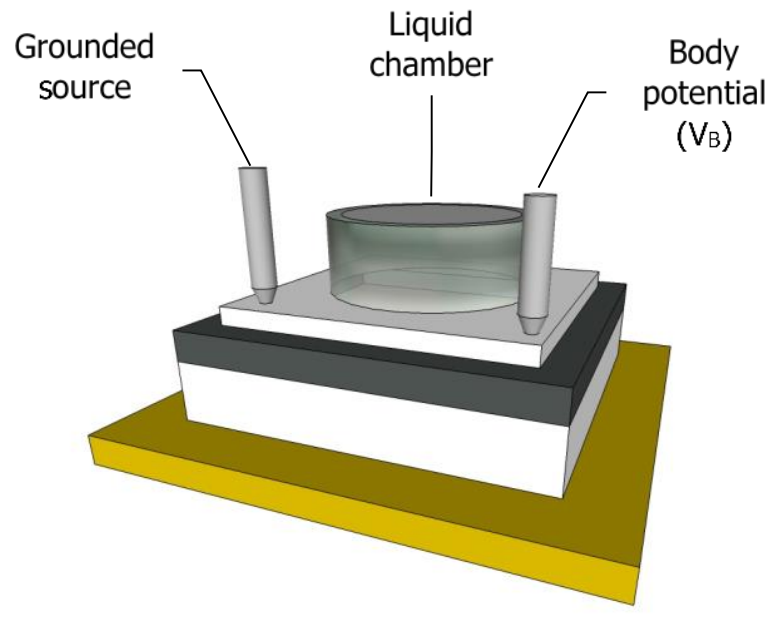

Figure 7: Schematic of the setup for biochemical sensing 
The body potential (Figure 8a) and the gate current (Figure 8b) are plotted against the gate voltage for three different setups: with a drain probe at $\mathrm{V}_{\mathrm{D}}$ equal to $0.1 \mathrm{~V}$ or $0 \mathrm{~V}$ or without drain probe. It can be noticed that the absence of the drain probe only results in a slight increase of the body potential (as well as the gate current peak). Indeed, the drain probe, when connected and biased, makes easier the evacuation of carriers injected in the film (during the accumulation or the inversion regime). The out-of-equilibrium effect is stronger and the measured $\mathrm{V}_{\mathrm{B}}$ exhibits higher values without the drain probe. This is very advantageous in the case of the biochemical sensing, because it simplifies the electrical setup.
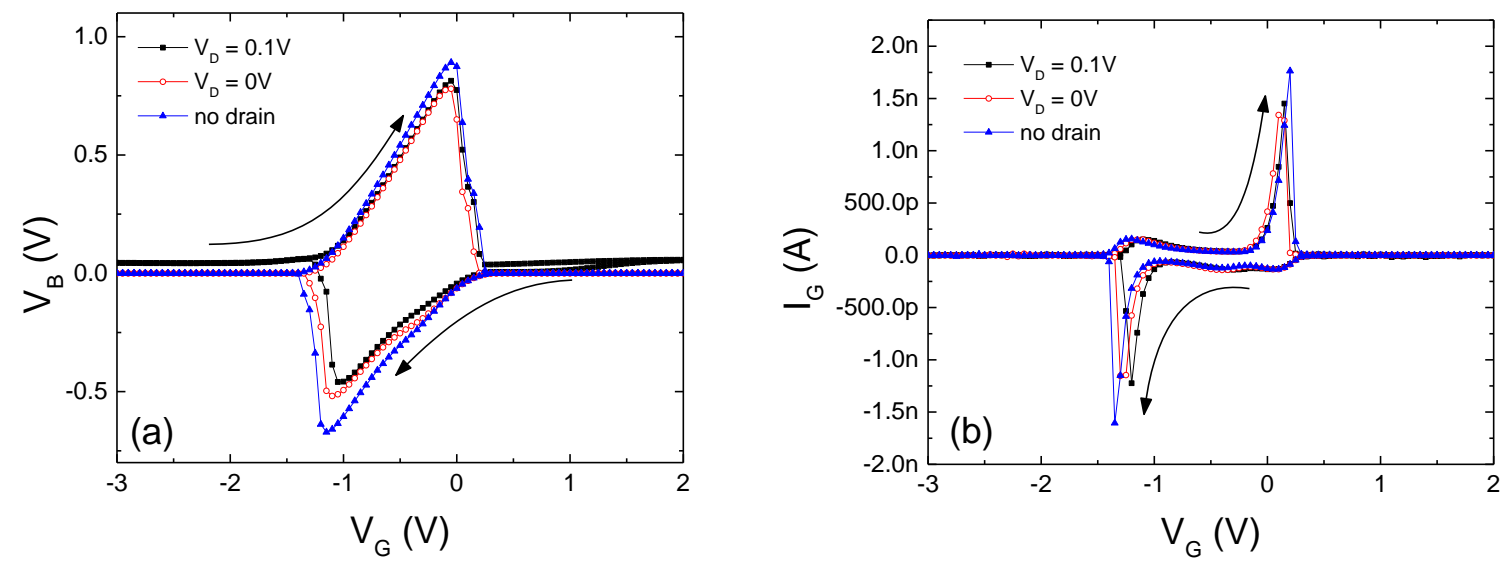

Figure 8: Body potential vs. gate voltage (a) and gate current vs. gate voltage (b) for different drain configurations. The configuration without a drain probe shows slightly higher values of $V_{B}$. 2 probe measurement, $V_{G}$ step $50 \mathrm{mV}$, probe pressure $100 \mathrm{~g}$.

The effect of the position of the probes was also investigated. The body potential measurement probe and the reference probe were placed in different positions of the $\Psi$-MOSFET island area: in the centre, on the edge and in the corner (Figure 9a). The measurements show no change in either $V_{B}$ or $I_{G}$ characteristics (Figure 9b). This fact is very favourable in the case of using a liquid chamber placed in the centre of the device, which necessitates contacts positioned outside of this region.

(a)
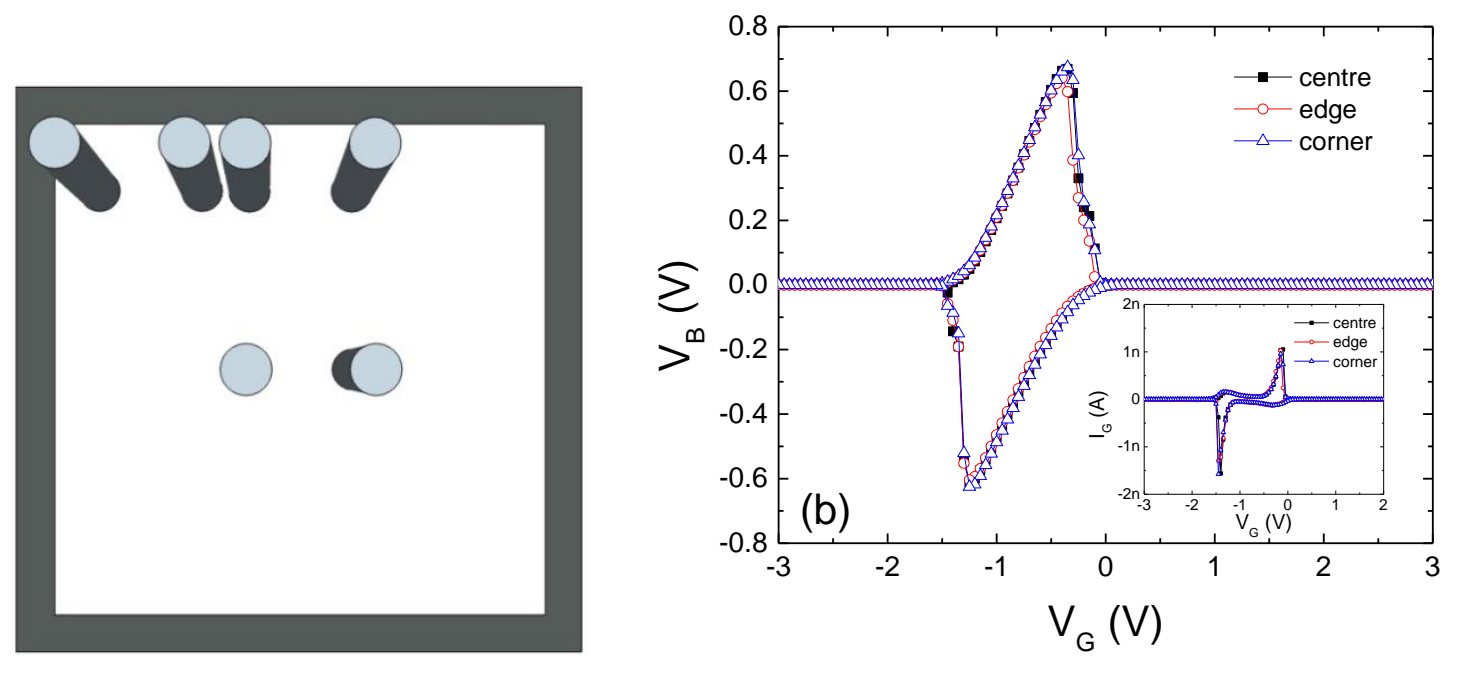

Figure 9: Probe position on the sample (a), body potential and gate current-in the inset - vs. gate voltage characteristics for different positions on the SOI surface (b). The body potential is not affected by the position of the probes on the sample. 2 probe measurement, $V_{G}$ step $50 \mathrm{mV}$, probe pressure $100 \mathrm{~g}$.

In the $\Psi$-MOSFET characterization, it is known that the $\mathrm{I}_{\mathrm{D}}$ measurements are affected by the distance between the probes [10].The influence of the probe distance on $V_{B}$ was also investigated. As illustrated in Figure 10a, $V_{B}$ has a slightly lower value, when the distance between the two probes diminished. This effect can be expected due to the fact 
that the reference probe is grounded, so a small potential drop can be expected. The same response can be observed for the $I_{G}$ peak, as the two are related.
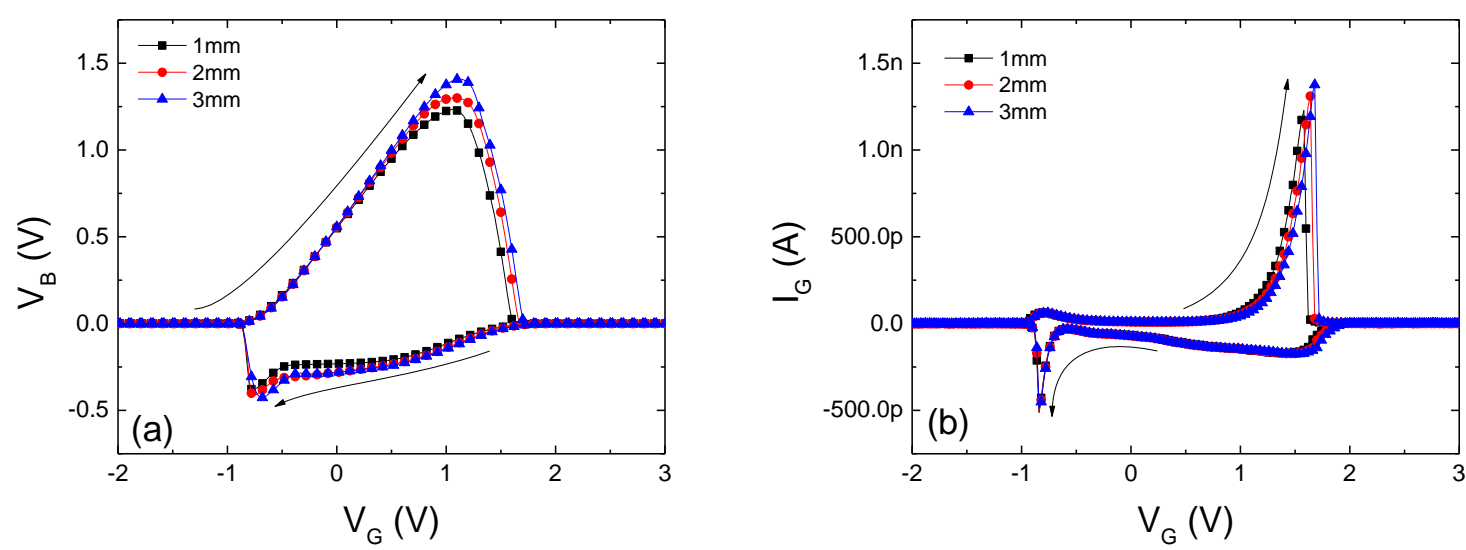

Figure 10: Body potential vs. gate voltage characteristics (a) and gate current vs. gate voltage characteristics (b). Both are slightly higher for a shorter distance between the probes. 2 probe measurement, $V_{G}$ step $50 \mathrm{mV}$, probe pressure $100 \mathrm{~g}$.

Additionally, the drain current is highly dependent on the pressure of the probes in the $\Psi$-MOSEFT configuration [9]. It is the stability of the $V_{B}$ sensing with respect to the applied pressure on the probes which will be subsequently validated. Figure 11 presents the results for different pressures applied to the body potential measuring probe (Figure $11 \mathrm{a}$ and $\mathrm{b}$ ) while the reference probe is maintained at $100 \mathrm{~g}$ or to the reference electrode (Figure $11 \mathrm{c}$ and $\mathrm{d}$ ) while the body potential probe is maintained at $100 \mathrm{~g}$. In all circumstances, the values of $\mathrm{V}_{\mathrm{B}}$ as well as $\mathrm{I}_{\mathrm{G}}$ are very similar and a small variation can be observed only in the region where $V_{B}$ reaches equilibrium. This proves that the body potential measurements are not influenced by the pressure applied on the probes.

All of the results in this section lead to the conclusion that the body potential measurements in the " $\Psi$-MOSEFT 2 probe configuration" provide a highly reliable sensing method due to its invariability with respect to the position, the distance and the pressure applied on the probes. 

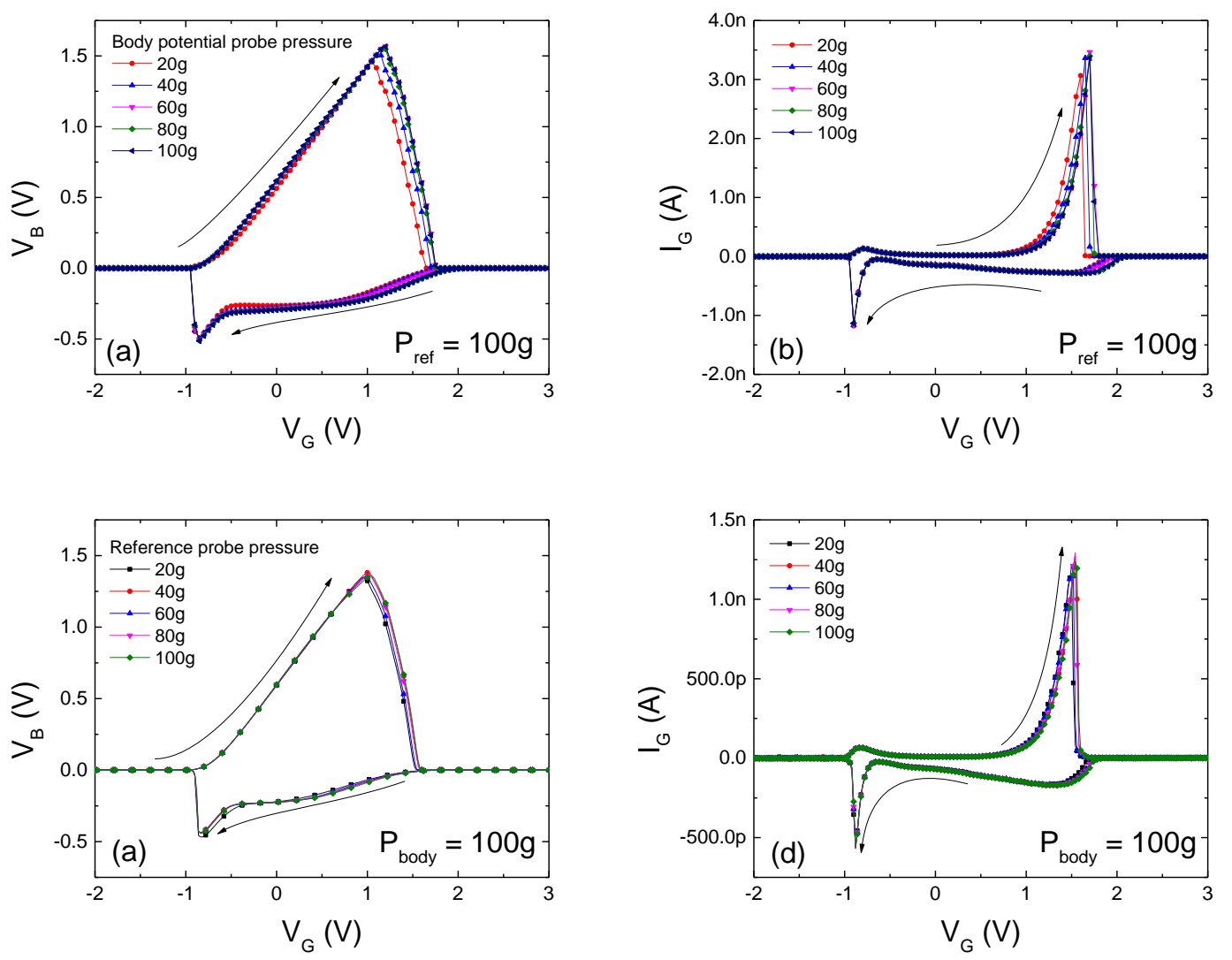

Figure 11: The influence of the pressure on the body potential probe $(a)$ and $(b)$, on the reference probe $(c)$ and $(d)$. The differences observed are negligible. 2 probe measurement, $V_{G}$ step $50 \mathrm{mV}$.

\section{Body potential for sensing}

The suitability of body potential measurements for sensing applications was verified on SOI samples after chemical treatments that are known to modify the charge on the top silicon film surface. Note that the samples are passivated with a thermal silicon dioxide grown on top of the silicon film, which is mandatory for the functionalization steps. The measurements were realized after an APTES ((3-Aminopropyl)triethoxysilane) treatment and gold nanoparticles (Au-np) attachment to the surface (process described in details in [11]). For APTES functionalization, the sample, cleaned with deionized water and ethanol, was exposed for 30 minutes to a diluted solution of APTES and was then dried at $120^{\circ} \mathrm{C}$ for 30 minutes on a hotplate. The gold nanoparticle adsorption was realized by covering the $\Psi$ MOSEFT device with a Au-np solution for 20 minutes, rinsing it with deionized water and then drying it with a nitrogen blower. In both cases, the chemical treatment modifies the top surface charge (positive after APTES and negative after Au-np deposition).

$\mathrm{I}_{\mathrm{D}}-\mathrm{V}_{\mathrm{G}}$ in the logarithmic and linear scale (inset) (Figure $12 \mathrm{a}$ ), $\mathrm{V}_{\mathrm{B}}-\mathrm{V}_{\mathrm{G}}$ (Figure $12 \mathrm{c}$ ), as well as $\mathrm{I}_{\mathrm{G}}-\mathrm{V}_{\mathrm{G}}($ Figure $12 \mathrm{~d}$ ) curves are shifted to the left after APTES, and to the right after gold nanoparticles deposition as expected, given the sign of the charge added to the surface. The shifts of $\mathrm{V}_{\mathrm{T}}, \mathrm{V}_{\mathrm{FB}}$ and $\mathrm{V}_{\mathrm{B}}$ are presented in Table 2 . The threshold voltage and the flat-band voltage were calculated using the $\mathrm{Y}$ function [12]. The values for the $\mathrm{V}_{\mathrm{B}}$ shifts are calculated at the point where the body potential returns to equilibrium. All the shifts are consistent, which means that the body potential can be used to evaluate the surface charge state. The observed $\Delta \mathrm{V}_{\mathrm{B}}$ shifts are higher than the variations observed for different probe positions on the same sample $(\sim 150 \mathrm{mV})$ or for different dies on the same SOI wafer $(\sim 300 \mathrm{mV})$. Additionally, for the $\mathrm{V}_{B}-\mathrm{V}_{\mathrm{G}}$ curve, the maximum response is obtained for a rather low gate voltage, while the $\mathrm{I}_{\mathrm{D}}$ needs to be observed at higher $\mathrm{V}_{\mathrm{G}}$. This is advantageous for sensing applications. 

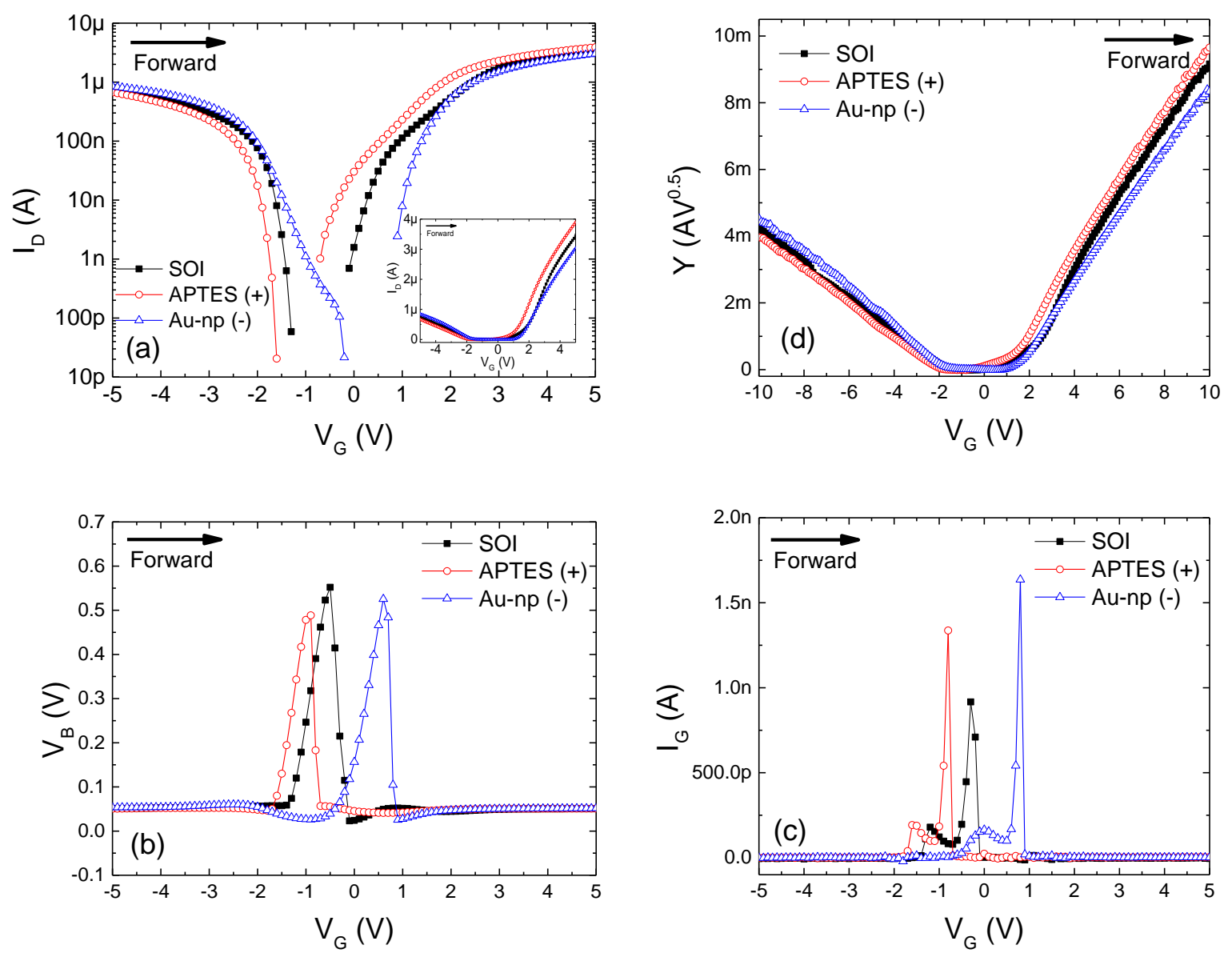

Figure 12: Drain current (a), Y-function (b), body potential (c) and gate current (d) vs. gate voltage for different chemical treatments of the SOI. The shifts between the steps are well correlated for the different parameters. 3 probe measurement, $V_{D}=0.1 \mathrm{~V}$, $V_{G}$ step $100 \mathrm{mV}$, probe pressure $100 \mathrm{~g}$.

Table 2: Electrical characteristics obtained before and after functionalization with APTES and Au-np.

\begin{tabular}{cccccc}
\hline Functionalization & $\mathbf{V}_{\mathbf{T}}(\mathbf{V})$ & $\boldsymbol{\Delta} \mathbf{V}_{\mathbf{T}}(\mathbf{V})$ & $\mathbf{V}_{\mathbf{F B}}(\mathbf{V})$ & $\Delta \mathbf{V}_{\mathbf{F B}}(\mathbf{V})$ & $\Delta \mathbf{V}_{\mathbf{B}}(\mathbf{V})$ \\
\hline Bare SOI & 0.54 & & -1.05 & & \\
APTES & 0.11 & -0.43 & -1.61 & -0.56 & -0.6 \\
Au-np & 0.88 & 0.76 & -0.50 & 1.11 & 1.0 \\
\hline
\end{tabular}

\section{Conclusion}

The research presented in this paper assesses the out-of-equilibrium body potential measurements applied to the $\Psi$ MOSFET configuration. In the first part, the correspondence between the main parameters was determined. It was determined that in the depletion region, where $I_{D}$ is negligible, $V_{B}$ is directly related to $V_{G}$ and that the $I_{G}$ peak is also observed in the derivative of $\mathrm{V}_{\mathrm{B}}$ with respect to $\mathrm{V}_{\mathrm{G}}$. The out-of-equilibrium nature of the body potential was exposed and the $\mathrm{I}_{\mathrm{G}}$ current was proved to be a displacement current responsible for the charging of the bottom interface of the BOX. Subsequently, a simplified version using only two probes of the previously presented setup was established and its advantages in terms of stability with respect to the position, the distance and the applied pressure on the probes were exposed. Ultimately, the proof of concept was made using APTES and Au-np functionalization.

The body potential monitoring is a promising sensing method due to its simplicity, sensitivity, the measurement of a potential instead of a current and the low bias needed for detection compared to the conventional drain current measurement. The body potential will be monitored in transient in order to assess our interpretations and for a broad range of applications based on charge detection (DNA detection, $\mathrm{pH}$ measurement),Acknowledgments Authors would like to thank Professor Sorin Cristoloveanu for inspiring comments and discussions. The authors thank also SOITEC for SOI wafers and Martine Gri, Aude Bouchard and Xavier Mescot for technical assistance during the 
sample preparation. This work was supported by the AGIR-POLE CLEPS project founded by the University of Grenoble Alpes and the european project WayToGoFast.

\section{Bibliography}

[1] S. Williams, S. Cristoloveanu, and G. Campisi, 'Point contact pseudo-metal/oxide/semiconductor transistor in as-grown silicon on insulator wafers', Mater. Sci. Eng. B, vol. 12, no. 1-2, pp. 191-194, 1992.

[2] S. Cristoloveanu, M. Bawedin, and I. Ionica, 'A review of electrical characterization techniques for ultrathin FDSOI materials and devices', Solid-State Electron., vol. 117, pp. 10-36, Mar. 2016.

[3] S. Cristoloveanu, D. Munteanu, and M. S. Liu, 'A review of the pseudo-MOS transistor in SOI wafers: operation, parameter extraction, and applications’, IEEE Trans. Electron Devices, vol. 47, no. 5, pp. 1018-1027, 2000.

[4] G. Hamaide, F. Allibert, H. Hovel, and S. Cristoloveanu, 'Impact of free-surface passivation on silicon on insulator buried interface properties by pseudotransistor characterization', J. Appl. Phys., vol. 101, no. 11, p. 114513, Jun. 2007.

[5] C. Fernandez, N. Rodriguez, C. Marquez, and F. Gamiz, 'Determination of ad hoc deposited charge on bare SOI wafers', in Ultimate Integration on Silicon (EUROSOI-ULIS), 2015 Joint International EUROSOI Workshop and International Conference on, 2015, pp. 289-292.

[6] M. Bawedin, S. Cristoloveanu, D. Flandre, and F. Udrea, 'Dynamic body potential variation in FD SOI MOSFETs operated in deep non-equilibrium regime: Model and applications', Solid-State Electron., vol. 54, no. 2, pp. 104-114, Feb. 2010.

[7] Hewlett-Packard Company, 'Semiconductor Parameter Analyzer User's Dictionary Reference'. 1995.

[8] S. Ruffell, J. E. Bradby, J. S. Williams, and O. L. Warren, 'An in situ electrical measurement technique via a conducting diamond tip for nanoindentation in silicon', J. Mater. Res., vol. 22, no. 3, pp. 578-586, Mar. 2007.

[9] I. Ionica, I. Savin, W. Van Den Daele, T. Nguyen, X. Mescot, and S. Cristoloveanu, 'Characterization of siliconon-insulator films with pseudo-metal-oxide-semiconductor field-effect transistor: Correlation between contact pressure, crater morphology, and series resistance', Appl. Phys. Lett., vol. 94, no. 1, p. 12111, Jan. 2009.

[10] K. Komiya, N. Bresson, S. Sato, S. Cristoloveanu, and Y. Omura, 'Detailed Investigation of Geometrical Factor for Pseudo-MOS Transistor Technique', IEEE Trans. Electron Devices, vol. 52, no. 3, pp. 406-412, Mar. 2005.

[11] I. Ionica, A. E. H. Diab, and S. Cristoloveanu, 'Gold nanoparticles detection using intrinsic SOI-based sensor', in Nanotechnology (IEEE-NANO), 2011 11th IEEE Conference on, 2011, pp. 38-43.

[12] G. Ghibaudo, 'New method for the extraction of MOSFET parameters', Electron. Lett., vol. 24, no. 9, p. 543, 1988. 